\author{
ARTIFICIAL SATELLITES, Vol. 53, No. 2 - 2018 \\ DOI: 10.2478/arsa-2018-0007
}

\title{
PERFORMANCE OF ABSOLUTE REAL-TIME MULTI-GNSS KINEMATIC POSITIONING
}

\author{
Kamil Kazmierski \\ Institute of Geodesy and Geoinformatics, \\ Wrocław University of Environmental and Life Sciences \\ C.K. Norwida 25, 50-357 Wrocław, Poland \\ e-mail: kamil.kazmierski@upwr.edu.pl
}

\begin{abstract}
Recently, we observe the rapid development of the Global Navigational Satellite Systems (GNSS), including autonomous positioning techniques, such as Precise Point Positioning (PPP). The GNSS have different conceptions, different spacecraft and use different types of orbits which is why the quality of real-time orbit and clock products is inconsistent, thus, the appropriate approach of the multi-GNSS observation processing is needed to optimize the solution quality. In this paper, the kinematic field experiment is conducted in order to examine multi-GNSS real-time Standard Point Positioning (SPP) and PPP performance. The test was performed on the $26 \mathrm{~km}$-long car route through villages, forests, the city of Wrockaw, crossing under viaducts and a high tension line. For the first time, the solution is based on GPS + GLONASS + Galileo + BeiDou observations using streamed corrections for orbits and clocks with two different weighting scenarios. Thanks to the usage of the multi-GNSS constellation the number of positioning epochs possible to determine increases by $10 \%$. The results show also that the appropriate weighting approach can improve the root mean square error in the SPP solution by about $13 \%$ and $42 \%$ for the horizontal and vertical coordinate components, respectively. In the case of PPP, the maximum quality improvement equals $70 \%$ for the horizontal component and the results for the vertical component are comparable with those obtained for the GPS-only solution.
\end{abstract}

Keywords: multi-GNSS, positioning, PPP, SPP, real-time corrections

\section{INTRODUCTION}

We have been witnessing the rapid development of the Global Navigational Satellite Systems (GNSS) since 1960, that is, when the first navigational satellites were launched. The current GNSS constellation, including GPS, GLONASS, Galileo, BeiDou, QZSS, and NavIC, consists of almost 100 spacecraft (Teunissen and Montenbruck 2017). Such a great number of navigational satellites allows for positioning in the challenging environment e.g. urban canyons (Groves 2011). Simultaneously with GNSS development, there was the necessity to provide high-quality clocks, orbits, and other products that support emerging systems. To fulfill this requirement, the Multi-GNSS Experiment (MGEX) was established by the International GNSS Service (IGS, (Dow et al. 2009)) to track, collate and analyze all available GNSS signals 
(Montenbruck et al. 2014). Unfortunately, the most precise products are available with the latency that makes it impossible to use them in real-time applications.

The real-time users have to apply products provided without a latency. One of the possibilities is to use the predicted part of the ultra-rapid MGEX solution. However, the main limitation of these products is clock predictions that are still a challenge (Martinez and Waller 2009). Another way is to apply real-time products dedicated to real-time applications and provided via RTCM (Radio Technical Commission for Maritime) streams (IGS 2015). Real-time products are provided with no charges by the IGS through IGS-Real-Time Service (IGS-RTS) or by IGS analysis centers (ACs) e.g. by Centre National d'Études Spatiales (CNES). The IGS-RTS provides official GPS and unofficial GLONASS products while streams provided by CNES contain products for the multi-GNSS constellation. Both streams contain precise clock and orbit corrections, while CNES additionally provides code and phase satellite biases as well as ionosphere maps, which allow performing the ambiguity resolution in real-time (Laurichesse 2011; Loyer et al. 2012). However, multi-GNSS corrections are not consistent and the quality of the satellite positions and clocks is different for various GNSS (Hadas and Bosy 2015; Kazmierski et al. 2018b). Thus, multi-GNSS processing with real-time streams needs an appropriate weighting approach to consider corrections' inconsistencies (Kazmierski et al. 2018a).

Nowadays, there exists a great need for having equipment with real-time positioning feature in everyday activities. GNSS sensors are a part of smart devices and allow for positioning with $10 \mathrm{~cm}$ accuracy in the fast-static surveys (Realini et al. 2017). One can distinguish the multitude of multi-GNSS applications, e.g., positioning of vehicles on the land (Knoop et al. 2017), on the sea (Lachapelle et al. 1987) and in the air (Rieke et al. 2011) precision agriculture (Tayari et al. 2015), the precise dynamic displacement detection (Paziewski et al. 2018) and monitoring performance of athletes (Aughey and Falloon 2010). Therefore, the improvement of the algorithms increasing the accuracy and the stability of positioning is expected.

One of the techniques which take advantage of real-time products is Precise Point Positioning (PPP) (Zumberge et al. 1997). PPP is an autonomous technique that does not need a dense global infrastructure as in the case of the Real-Time Kinematic (RTK) technique (Xu 2016). This feature allows for using PPP in many applications for which traditional RTK is limited because of too long distance to a reference station. There is a Wide Area RTK (WARTK) for which a distance between base and rover station can be extended but it is still a concept (Hernández-Pajares et al. 2004). Among applications of PPP, one can distinguish positioning, tsunami warning systems, and water vapor monitoring systems. Nevertheless, PPP is burdened with some drawbacks. One of the major limiting factors is the solution convergence time which can reach even several hours depending on a positioning method used (Héroux et al. 2004; Bisnath and Gao 2009; Kouba 2015).

Many of the current works evaluate kinematic positioning for the observations collected by the static stations (Tang et al. 2017; Wang et al. 2018), so they do not reflect positioning in the actual kinematic conditions. Actual kinematic data were used only to evaluate the performance of selected GNSS or relative positioning techniques (Geng et al. 2010; He et al. 2014; Yang et al. 2017). Former works show that the quality of the horizontal coordinate components is improved by $20 \mathrm{~mm}$ when using four GNSS system kinematic PPP solutions with final MGEX products (Guo et al. 2018). However, final MGEX products are not available for real-time purposes. In this paper, the real-time approach using streamed orbit and clock corrections is tested for the actual data collected by a precise multi-GNSS receiver being on the move. Quad constellation solution with GPS, GLONASS, Galileo and BeiDou dual-frequency data is evaluated combined with the real-time orbit and clock corrections from CNES. The test is 
conducted in the casual road conditions and with typical obstacles possible to meet during car driving.

\section{DATA AND METHODS}

\subsection{CASE STUDY}

In order to check the positioning quality while using multi-GNSS constellation, an experiment with the vehicle in motion was performed. A car was adopted as a moving platform with multi-GNSS Trimble R10 receiver installed on the roof. The test took place on January 5th, 2018 from 18:45 to 21:10. Thanks to late daytime selection the road traffic was rather calm, thus the route was smooth. The field test was divided into two parts. The first one took about $105 \mathrm{~min}$ in the open area without any movements in order to initialize the PPP solution. Then, the test in motion started and took about $35 \mathrm{~min}$ on the $26 \mathrm{~km}$ distance between Oleśnica and Wrocław in south-west Poland.

Figure 1 illustrates in detail the test route. The test route ran initially in the open space; there were $2 \mathrm{~km}$ long forest and small villages on the way. The density of the infrastructure increased with the distance and reached the maximum in Wrocław. Additionally, a high-tension $400 \mathrm{kV}$ line crossed the route at the point of about $4 \mathrm{~km}$ from the start. There were also five viaducts at the entrance to Wrocław (after $19 \mathrm{~km}$ ). Satellite visibility and DOP (Dilution of Precision) parameters are presented in Figure 2. During the test drive the number of satellites when using 4 systems is always at the minimum level of 8 satellites, but when using GPS-only it decreases to 4 . DOP parameters represent car surroundings and the instantaneous horizontal coverage. The presence of obstacles, such as a forest, viaducts and tall buildings, are represented by a bigger DOP which reaches more than 10 for some epochs.

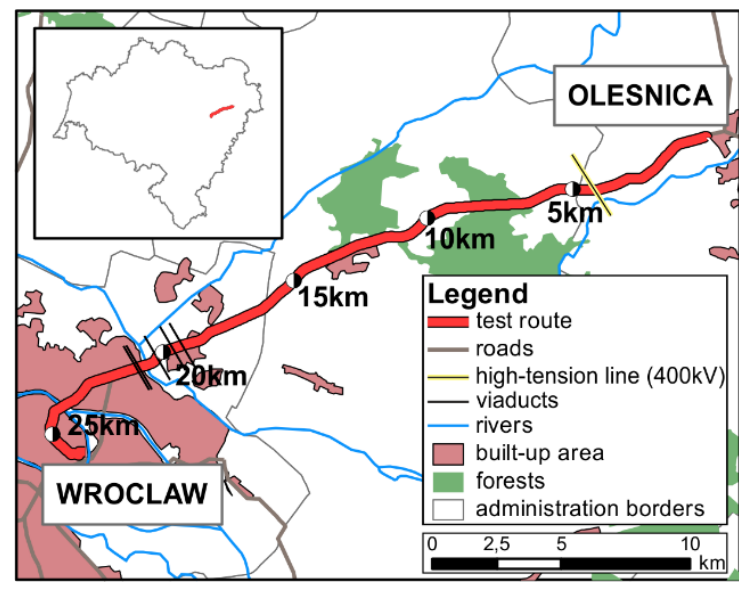

Fig. 1. Car route used for the kinematic test 


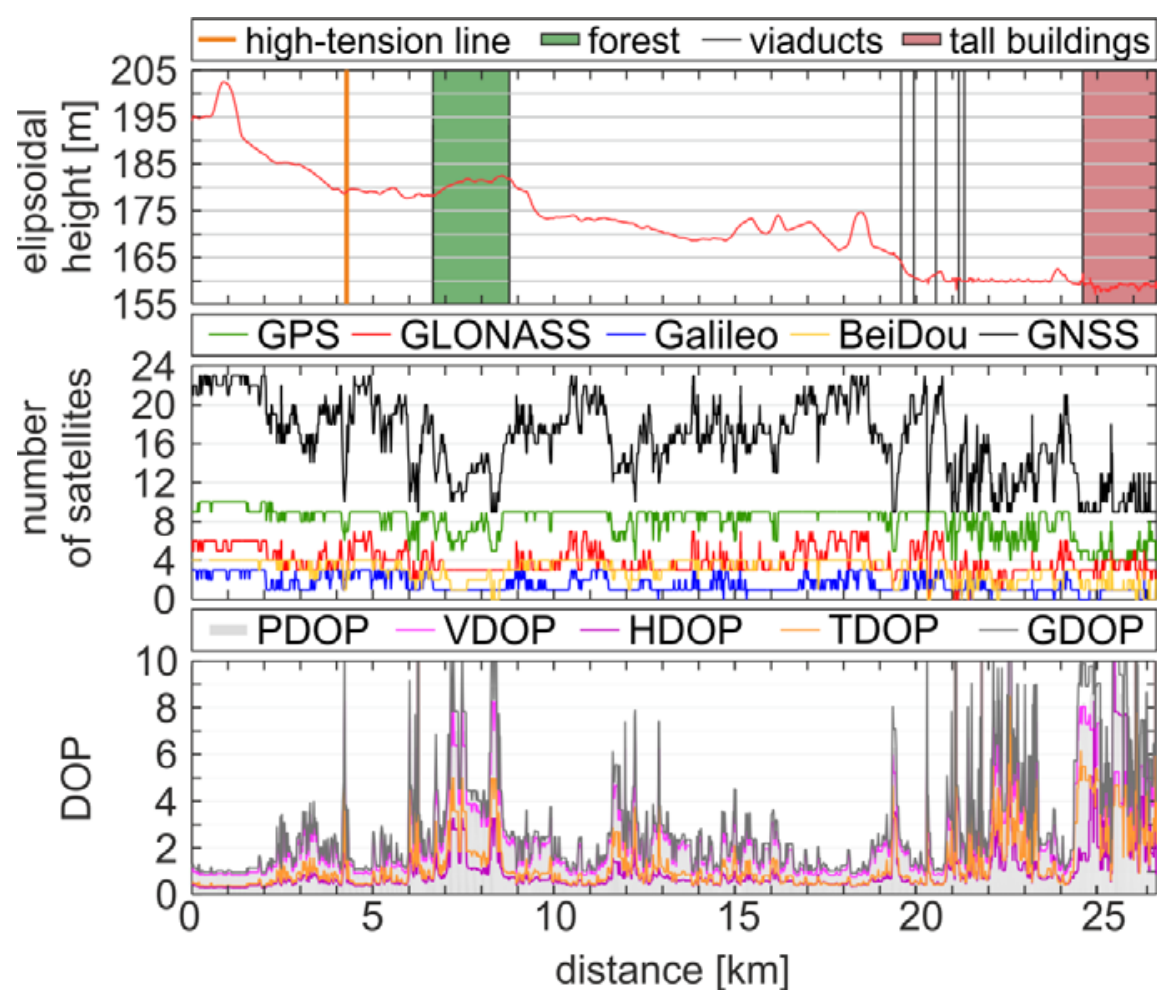

Fig. 2. Elevation profile, number of satellites and DOP parameters for the kinematic test route in reference to the starting point of the road experiment.

The reference positions of the receiver are computed in RTKlib v 2.4.2 (Tomoji 2007) based on final orbits using the RTK solution which is applicable for distances up to $20 \mathrm{~km}$ (Rietdorf et al. 2006). The final MGEX products provided by the Center for Orbit Determination in Europe (CODE, Prange et al. 2017) are used for the reference solution. The nearest permanent GNSS stations are located in Wrocław (WROC) and in Oleśnica (OLES) and the distance between them is equal to $24 \mathrm{~km}$. Thus, the reference solution is a combination of two RTK solutions for those reference stations. In order to eliminate the uncertainty about the reference solution, float solutions are eliminated from the reference coordinate set and only solutions with fixed resolved RTK ambiguities are used. For both stations, the kinematic trajectory was computed using L1+L2 data with the broadcast ionosphere model and Sastamoinen troposphere model. The differences between solutions were below $3 \mathrm{~cm}$. The final reference solution is a mean value of the positions computed with a reference to WROC and OLES weighted by the distance to the reference station. The reference stations' positions are computed as a daily PPP solution using final CODE products. Thus the RTK solution is computed in the ITRF 2014 at the epoch of the measurements and is consistent with real-time PPP solutions.

\subsection{SOLUTION STRATEGY}

The kinematic trajectory is computed using actual GPS, GLONASS, Galileo and BeiDou observations in a common least square adjustment. Observations are processed using in-house-developed GNSS-WARP (Wrocław Algorithms for Real-Time Positioning) software (Hadas 2015). Additionally, to account for the antenna phase center correctly, the absolute IGS antenna correction igs 14. atx file is used. The Antenna Exchange Format (ANTEX) file contains calibration information for GPS and GLONASS for two frequencies. In order to fill the gap for the remaining systems, the offsets from GPS and GLONASS are adopted for Galileo and BeiDou using the nearest frequencies.

Data are processed in six different variants, being a combination of two strategies and three inter-system weighting scenarios, defined as follows. The first strategy, namely SPP (Standard 
Point Positioning) is based only on code observations, whereas the second strategy is based on the PPP technique with code and carrier-phase observations. Both strategies take advantage of the real-time corrections using exactly the same supplementary information and having the same software settings. The only exception is handling of the troposphere delay. In SPP total delay is fixed to the UNB3 model, while in PPP the non-hydrostatic part is estimated. All processing parameters are presented in Table 1. The weight matrix in the least squares adjustment is defined as a diagonal square matrix with diagonal elements equal $\mathrm{P}_{\mathrm{ii}}=1 / \sigma_{\mathrm{ii}}{ }^{2}$ where $\sigma$ denotes the standard deviation of a priori error of the $i^{\text {th }}$ observation. Additionally, three weighting scenarios are tested for each strategy. The first scenario, called "GPS-only" further in this paper, is the GPS only solution with weights calculated using $\sigma$ of $0.300 \mathrm{~m}$ and $0.010 \mathrm{~m}$ for code pseudoranges and carrier-phase observations, respectively. "Equal" scenario is a multi-GNSS solution with equal weights for each system corresponding to weights from the GPS-only scenario. "GxS" scenario is a multi-GNSS solution with individual weights for each system (Kazmierski et al. 2018a) that take into account the quality of real-time corrections (Table 2).

Table 1 Summary of the processing strategy

\begin{tabular}{|c|c|}
\hline Observables & ionosphere-free pseudorange and carrier-phase \\
\hline \multirow[t]{2}{*}{ Ambiguities } & float (not fixed) \\
\hline & GPS: L1/L2, \\
\hline Frequencies (RINEX 3.03 & GLONASS: G1/G2, \\
\hline notation) & Galileo: E1/E5, \\
\hline & BeiDou: B1/B2 \\
\hline Elevation weighting & elevation (e)-dependent weighting: $\sin (e)$ \\
\hline Intra-system weighting & $\begin{array}{l}\sigma=0.300 \mathrm{~m} \text { for code, } \sigma=0.010 \mathrm{~m} \text { for carrier-phase in } \\
\text { variants "GPS-only" and "Equal" (see (Kazmierski et al. } \\
2018 \mathrm{a} \text { ) for more details) }\end{array}$ \\
\hline Inter-system weighting & Equal: equal weights, GxS: see Table 2 \\
\hline Elevation cut-off angle & $5^{\circ}$ \\
\hline Sampling rate & $0.2 \mathrm{~s}$ \\
\hline Troposphere delay modeling & $\begin{array}{l}\text { UNB3m mapping functions (Leandro et al. 2008) and } \\
\text { a priori value for the hydrostatic delay, wet delay } \\
\text { estimated as } 4 \mathrm{~mm} / \sqrt{\text { hour random walk process (Hadas et }} \\
\text { al. 2017) }\end{array}$ \\
\hline Receiver clock & estimated as white noise, individual clock for each GNSS \\
\hline Satellite orbits and clocks & fixed from real-time CNES stream (mountpoint CLK93) \\
\hline Code and phase biases & $\begin{array}{l}\text { absolute, from real-time CNES stream (mountpoint } \\
\text { CLK93) in BKG NTRIP Client format (BNC, v 2.12, } \\
\text { (Weber and Mervart 2009)). }\end{array}$ \\
\hline Solution type & static/kinematic with float ambiguities \\
\hline Correction models & $\begin{array}{l}\text { phase wind-up, relativistic delays, solid earth tides, } \\
\text { receiver antenna phase center offset and variation(Petit } \\
\text { and Luzum 2010) }\end{array}$ \\
\hline
\end{tabular}

$\overline{B K G \text { (Federal Agency for Cartography and Geodesy), NTRIP (Networked Transport of RTCM }}$ via Internet Protocol) 
Table 2. Pseudorange code and carrier phase standard deviations $\sigma$ used for calculating weights in the processing variant "GxS", for details see (Kazmierski et al. 2018a).

\begin{tabular}{lccc}
\hline & & Pseudorange code $[\mathrm{m}]$ & Carrier-phase $[\mathrm{m}]$ \\
\hline GPS & & 0.300 & 0.0100 \\
GLONASS & & 0.852 & 0.0284 \\
Galileo & & 0.964 & 0.0321 \\
\hline \multirow{2}{*}{ BeiDou } & GEO & 10.876 & 0.3625 \\
GEO & IGSO & 1.199 & 0.0400 \\
& MEO & 1.088 & 0.0363 \\
\hline
\end{tabular}

The series of receiver positions were computed in the simulated real-time mode, in order to replicate the same processing conditions for each variant. The processing mode is set depending on the car movement. During the initialization time, the position is computed in the static mode, whereas as soon as the car starts to move, the processing is switched to the kinematic mode.

\section{RESULTS}

Results from 6 processing variants are assessed on the basis of the number of the computed epochs for the particular solution. Additionally, the quality of the computed coordinates is evaluated by analyzing the root mean square errors (RMSE) with respect to the reference solution. In the RMSE comparison solutions from common epochs are considered. Analysis results are provided separately for the horizontal $(\mathrm{Hz})$ and the vertical (U) components.

\subsection{SOLUTION AVAILABILITY}

A number of epochs with computed coordinates is the first measure that can describe the advantage of the multi-GNSS solution. During the test route, 10210 epochs of observation were recorded. The number of properly computed epochs is different among tested variants as listed in Table 3. The differences are mainly visible when the satellite visibility is limited due to the terrain obstacles - in the forest and from the viaducts to the end of the track. The lack of a solution in PPP is connected with the low observation quality, whereas in SPP is connected with the low number of satellites being visible. Observations are affected by cycle slips or are burdened with strong multipath effect, and as a consequence, those observations are detected as outliers. In the conducted experiment the multi-GNSS constellation increases the number of properly computed epochs by about $15 \%$ when compared to the GPS-only solution. The gain of solution epochs is possible because of a greater number of visible satellites in the challenging environment with many trees and building limiting the reception of satellite signals during the test drive.

Table 3. The number of the computed epochs for different solutions.

\begin{tabular}{llccc}
\hline system & $\begin{array}{c}\text { weighting } \\
\text { scenario }\end{array}$ & $\begin{array}{c}\text { computed } \\
\text { epochs }\end{array}$ & $\begin{array}{c}\text { computed } \\
\text { epochs [\%] }\end{array}$ \\
\hline \multirow{2}{*}{ PPP } & GPS-only & Equal & 8178 & 80.1 \\
& multi-GNSS & Equal & 9401 & 92.1 \\
& multi-GNSS & GxS & 9373 & 91.8 \\
\hline \multirow{2}{*}{ SPP } & GPS-only & Equal & 8826 & 86.4 \\
& multi-GNSS & Equal & 9684 & 94.8 \\
& multi-GNSS & GxS & 9684 & 94.8 \\
\hline
\end{tabular}


The increase of the computed epochs is not related to the weighting scenario. The number of computed epoch differences between GxS and Equal scenario is below 1\%. Therefore, in order to increase the number of solutions, more than one system should be used. The weighting scenario does not significantly affect the quality control procedure, though. Moreover, the number of the computed epochs is different in SPP and PPP solutions. The differences may be caused by the software quality control module and the cycle slips occurrence for the carrier-phase observations.

\subsection{QUALITY OF CODE PSEUDORANGE ONLY POSITIONING: STANDARD POINT POSITIONING}

Figure 3 shows the time series of coordinate residuals for the East, North and Up components with respect to the reference RTK solution. The obtained coordinate residuals for East and North components are at the comparable level for all epochs. Significantly larger residuals for horizontal components occur during the last part of the test route when tall buildings strongly cover signals from GNSS satellites. Horizontal coordinates are biased by $0.87 \mathrm{~m}$ for GPS-only and equal weighting schemes, while for GxS scenario the mean bias is $0.77 \mathrm{~m}$. The Up component is significantly biased for all weighting scenarios, with the most extreme value reaching $-2.3 \mathrm{~m}$ in the GPS-only scenario.
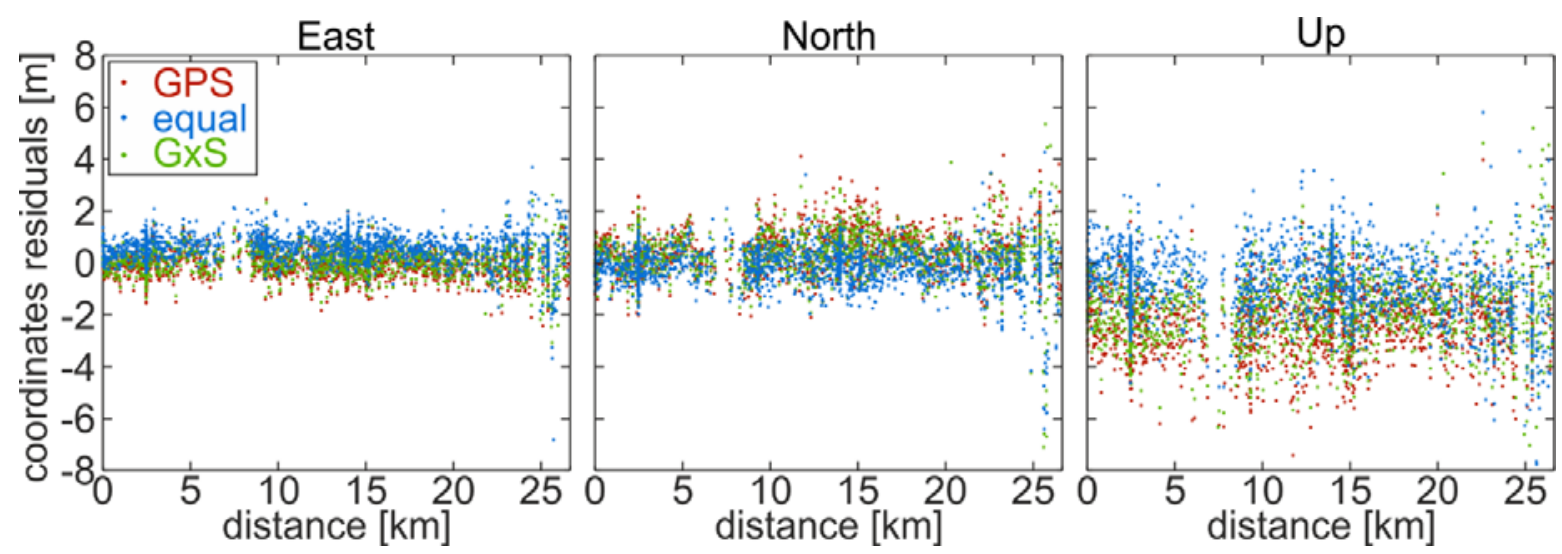

Fig. 3. Coordinate residuals for SPP variant for the kinematic experiment route for the East, North and Up components, respectively, with respect to the RTK solution

Table 4 summarizes the obtained quality of coordinates showing the RMSE for all weighting schemes. The significant improvement of the horizontal components for the multi-GNSS constellation is obtained only for the GxS weighting approach and the RMSE of coordinate residuals is reduced by $13 \%$ with respect to the GPS-only solution. In the case of the Up component, using the multi-GNSS constellation improves the quality in both tested scenarios. The smallest RMSE for the U component is reached for equal weights and is reduced by $42 \%$ when compared to the GPS-only scenario.

Table 4. RMSE of the SPP coordinate residuals in reference to the RTK solution for the kinematic experiment route for the horizontal and vertical components

\begin{tabular}{lcc}
\hline & \multicolumn{2}{c}{ RMSE $[\mathrm{m}]$} \\
\hline & $\mathrm{Hz}$ & $\mathrm{U}$ \\
\hline GPS-only & 1.02 & 2.59 \\
Equal & 0.98 & 1.50 \\
GxS & 0.89 & 2.17 \\
\hline
\end{tabular}




\subsection{PRECISE POINT POSITIONING QUALITY}

In opposition to SPP, in this variant, processing contains all epochs including epochs collected during the car stop at the beginning of the test drive. This stop allows for initialization of the solution. Figure 4 illustrates the time series of coordinate residuals for the East, North and Up components. During first $4 \mathrm{~km}$, the trajectory in reference to RTK positions is very stable. After the $4^{\text {th }} \mathrm{km}$, some discontinuities appear which are probably caused by the fact that the car route crosses the high-tension line $(400 \mathrm{kV})$. These discontinuities are not present in the RTK solution but a float solution appears for a single epoch. After this event, the quality of the solution is degraded and the coordinates are shifted. After crossing under the first viaduct, which is $19.6 \mathrm{~km}$ away from the start, the coordinate residuals reach up to $5 \mathrm{~m}$, because phase ambiguities are reinitialized due to cycle slips. The same happens around $24.8 \mathrm{~km}$ from the start when the car passes a very narrow street with tall buildings along it.

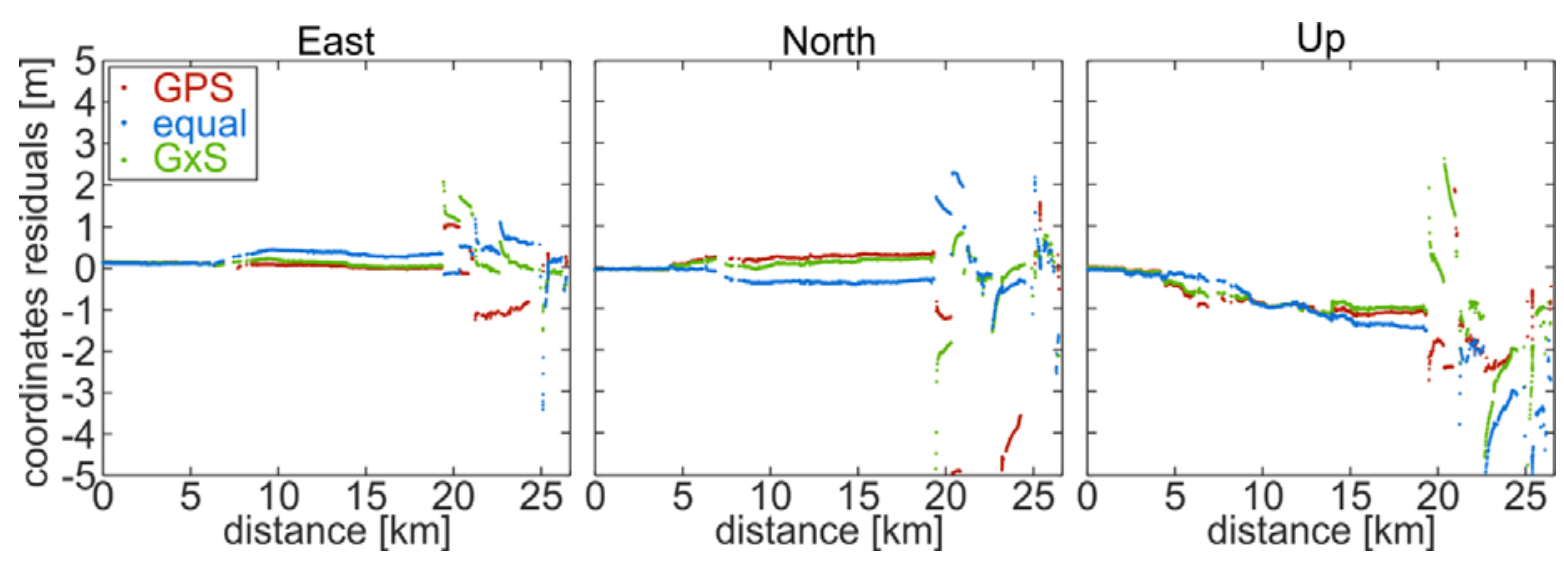

Fig. 4. Time series of coordinate residuals for PPP variant for the kinematic experiment route for the East, North and Up components, respectively, in reference to the RTK solution

Table 5 summarizes the RMSE of coordinate residuals in common epochs for each solution. The horizontal residuals for multi-GNSS scenarios are significantly lower than for the GPS-only scenario. The multi-GNSS solution is at least 3 times better than GPS-only. In the case of the Up component, the multi-GNSS brings a slight improvement (of about 2\%) only for the GxS weighting scenario. The equal weights applied to all systems degrade the solution more than twice when compared to GxS.

Table 5. RMSE of the PPP coordinate residuals in reference to the RTK solution for the kinematic experiment route for horizontal and vertical components, respectively

\begin{tabular}{lcc}
\hline & \multicolumn{2}{c}{ RMSE [m] } \\
\hline & $\mathrm{Hz}$ & $\mathrm{U}$ \\
\hline GPS-only & 1.80 & 1.07 \\
Equal & 0.61 & 2.34 \\
GxS & 0.54 & 1.05 \\
\hline
\end{tabular}

\subsubsection{INITIALIZATION PERIOD}

The first static part of the field test is used as the time needed for the initialization of the PPP solution. Data stored during the first 105 min of the test allow also for proving the correctness of the applied computation algorithm. It is confirmed that the applied algorithms work properly. The coordinate residuals in reference to the RTK solution for the initialization period in the local East, North and Up coordinate residuals are equal to $15.3,-5.7$ and $1.2 \mathrm{~cm}$, respectively. 
The formal errors of these coordinates equal to $0.2,0.1$ and $0.2 \mathrm{~cm}$ for East, North and Up components, respectively.

\subsubsection{FIRST SECTION OF THE ROUTE}

The residuals are analyzed from the starting point to the crossing with the high-tension line in order to emphasize the potential of the multi-GNSS PPP. The coordinate residuals obtained for the first $4 \mathrm{~km}$ of the route are shown in Figure 5. A short unscheduled maintenance stop of $3 \mathrm{~min}$ is visible at the distance of $2.5 \mathrm{~km}$. The results obtained for the GPS-only and for GxS scenario are very consistent and are about $1.5 \mathrm{~cm}$ more biased than the results from the Equal scenario, especially for the East component. The situation is opposite for the Up component for which Equal scenario assumes a bigger bias of about $4 \mathrm{~cm}$. However, the RMSE of differences for horizontal components are very small and their maximum values are equal to $2 \mathrm{~cm}$. The RMSE differences for the Up component for the Equal scenario are larger by $60 \%$ when compared to the GPS-only solution. Although the GxS scenario performs worse than the Equal scenario in the horizontal components and worse than the GPS-only solution for the Up component, it reduces the bias of the horizontal component and deteriorates the bias in the vertical position insignificantly when compared to the GPS-only solution.

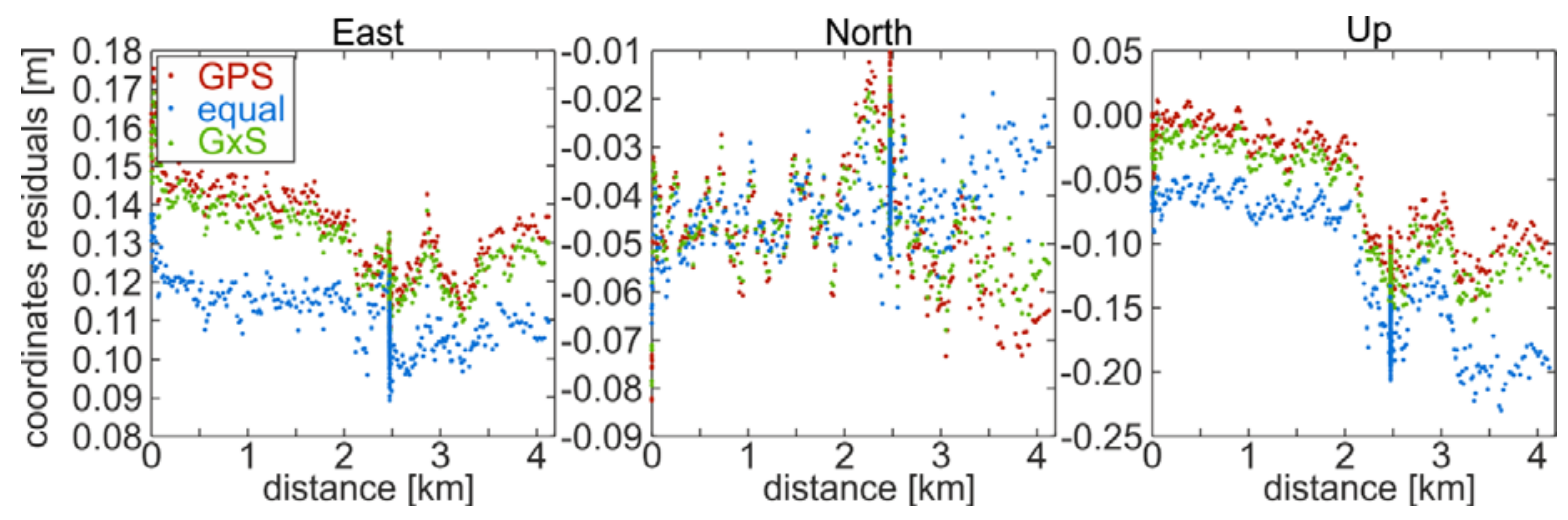

Fig. 5. Coordinate residual time series for PPP variant for the kinematic experiment route for the East, North and Up components, respectively, in reference to RTK solution

Table 6. RMSE of the PPP coordinate residuals in reference to the RTK solution for the first section of the route (from the starting point to the high-tension line) for horizontal and vertical components

\begin{tabular}{lcc}
\hline & \multicolumn{2}{c}{ RMSE [m] } \\
\hline & $\mathrm{Hz}$ & $\mathrm{U}$ \\
\hline GPS & 0.14 & 0.09 \\
Equal & 0.12 & 0.15 \\
GxS & 0.13 & 0.11 \\
\hline
\end{tabular}

\subsubsection{SECOND SECTION OF THE ROUTE}

The $15 \mathrm{~km}$ long track section between the high-tension line and the first viaduct has very consistent coordinate residuals for all tested scenarios which are summarized in Table 7. The high-tension line causes some problems in the GNSS solutions which are probably connected with the cycle-slip occurrence. Due to this event, a discontinuity appears in the residual time series. Furthermore, the $2 \mathrm{~km}$ long forest section degrades the solution even to a greater extent than the high-tension line (Hu et al. 2016). Coordinates from this section are much more biased than in the first section of the route. The RMSE for the horizontal coordinate residuals increases by $85 \%, 38 \%$, and $300 \%$ for GPS-only, GxS, and the Equal scenario, respectively, when compared the second section to the first section. This shows that the obstacle occurrence on the 
way introduces some problems, which need further investigations. Nevertheless, additional satellites from the multi-GNSS constellation with the appropriate weighting scenario prevent substantially from the degradation of the solution and give accuracy better than $20 \mathrm{~cm}$. On the other hand, coordinate residuals for the Up component increase significantly for all scenarios and are close to $1 \mathrm{~m}$. For the Up component, the results for GxS scenario bring the smallest RMSE, similarly as in the case of the horizontal components.

Table 7. RMSE of the PPP coordinate residuals in reference to RTK solution for the second section of the route (from the high-tension line to the first viaduct) for horizontal and vertical components

\begin{tabular}{lcc}
\hline & \multicolumn{2}{c}{ RMSE [m] } \\
\hline & $\mathrm{Hz}$ & $\mathrm{U}$ \\
\hline GPS & 0.26 & 0.98 \\
Equal & 0.48 & 1.09 \\
GxS & 0.18 & 0.90 \\
\hline
\end{tabular}

\subsubsection{THIRD SECTION OF THE ROUTE}

The last $7 \mathrm{~km}$ long section from the first viaduct to the end of the test route is evaluated separately because of the significant position degradation caused by the multitude of obstacles in the urban area. In total, 5 viaducts caused cycle slips and reinitialization of ambiguities, therefore the obtained coordinate residuals dramatically increase, because the processing software is not capable of the fast ambiguity recovery. For this section, RMSE values are listed in Table 8. Low satellite visibility appears in this section very often and the computed RMSE are several times bigger than in the previous sections. Nevertheless, multi-GNSS horizontal results are 4 times more accurate than the results obtained only with GPS satellites. The RMSE of the Up component in the GxS scenario remains at the level comparable to that of the GPS-only scenario.

Table 8. RMSE of the PPP coordinate residuals in reference to RTK solution for the third section of the route (from the first viaduct to the end of the test route) for horizontal and vertical components

\begin{tabular}{lcc}
\hline & \multicolumn{2}{c}{ RMSE [m] } \\
\hline & $\mathrm{Hz}$ & $\mathrm{U}$ \\
\hline GPS & 4.48 & 1.98 \\
Equal & 1.22 & 5.49 \\
GxS & 1.29 & 2.02 \\
\hline
\end{tabular}

\subsubsection{SUMMARY RESULTS FOR THE TEST ROUTE}

The presented results show the level of the accuracy possible to obtain with SPP and PPP techniques using the multi-GNSS constellation. Figure 6 summarizes the obtained RMSE for all calculation approaches. The coordinate residuals computed with PPP are smaller when compared to SPP but the horizontal components for GPS-only scenario and the Up component for Equal scenario bring surprisingly bigger RMSE by $1.1 \mathrm{~m}$ and $1.46 \mathrm{~m}$, respectively. However, while excluding the last part of the test track the results obtained with PPP are much more accurate. 

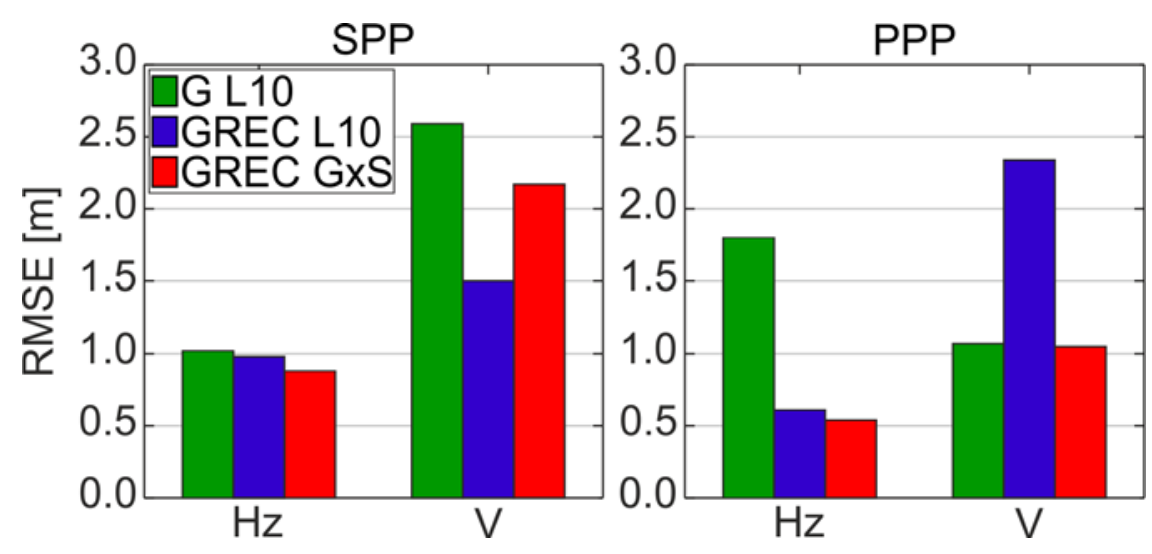

Fig. 6. RMSE of the SPP and PPP coordinate residuals in reference to RTK solution for the common epochs for horizontal and vertical components

\section{DISCUSSION}

The conducted experiments evaluate two autonomous positioning techniques: SPP and PPP using actual GNSS observations collected by the receiver installed on the roof of the car. The test track of $26 \mathrm{~km}$ length has various types of the surrounding: a high-tension line, viaducts, forest, and high buildings inside the city of Wrocław. All computations were performed using real-time products and the obtained results may also determine the level of accuracy possible to get when taking advantage of the freely available real-time clock and orbit corrections. Additionally, various accuracy levels for the presented weighting scenarios indicate that stochastic modeling is very important during multi-GNSS observation processing. The presented outcomes show that the application of improper weighting scenario when using more than one navigation system may lead to solution degradation and bring 4 times bigger RMSE.

A large number of the navigational satellites has a very positive contribution through providing a better satellite geometry. This fact allows for obtaining a position in a fast-changing environment with a substantial horizon coverage by buildings, trees, trucks etc. The gain caused by the signals from additional satellites introduced to the equation system increases the number of epochs with successfully computed positions by about $10 \%$.

The accuracy level possible to obtain using SPP supported by real-time products equals $1.02,0.98$ and $0.88 \mathrm{~m}$, for GPS-only, multi-GNSS with equal weights, and multi-GNSS GxS scenario, respectively. The impact of additional GNSS allows for reducing the RMSE of coordinate horizontal residuals by $12 \%$. This reduction was possible only for the GxS variant which considers inconsistencies of the real-time correction qualities. Equal weights for each system reduce the RMSE by 4\%. Considering the Up component, the biggest reduction of the coordinates residuals is for the Equal scenario and the profit is $42 \%$ while for $\mathrm{GxS}$ this reduction is $12 \%$.

In order to show the benefit from multi-GNSS real-time PPP, the test route is divided into 3 sections. Multi-GNSS positioning quality in the open area when using GxS scenario is comparable to the quality possible to be obtained with GPS-only and Equal scenarios. The advantage of the modified weighting scenario $(\mathrm{GxS})$ is observable in the more challenging environment (from the point of a high-tension line and a forest). GxS scenario allows the horizontal component RMSE to remain below $20 \mathrm{~cm}$. The quality of the Up component is visibly worse but still, GxS scenario brings the lowest RMSE. Tall buildings and viaducts cause a position degradation, thus the horizontal solution quality is about $1 \mathrm{~m}$ and $2 \mathrm{~m}$ for the Up component. This result is much better than the result obtained from the GPS-only solution for 
which RMSE reaches $4.5 \mathrm{~m}$. The previous experiment with real-time kinematic PPP considering GPS and GLONASS indicated that the quality of positioning while compared to RTK solution was at the level of $15-20 \mathrm{~cm}$ and $20-30 \mathrm{~cm}$ for horizontal and vertical components, respectively (Martín et al. 2015). Additionally, the obtained results increase results much more than $20 \mathrm{~mm}$ obtained in (Guo et al. 2018) what may be connected with used weighting scenarios adopted to the clock and orbit corrections quality applied in the processing. The accuracy of the GxS test scenario with regard to aforementioned work is better or at the comparable level for horizontal coordinates. The vertical coordinate is at least 2 times more accurate than in the cited work but the influence of a high-tension line probably affects the RMSE for this component significantly (Hu et al. 2016).

The PPP computations indicate some problems with terrain obstacles encountered on the car way. Among these obstacles a high-tension line can be distinguished which causes cycle slips and, as a consequence, a coordinate discontinuity. The road infrastructure, such as viaducts, may affect the results as well. Additionally, buildings and natural elements, such as forests, also disturb the reception of the GNSS signal. Elimination of the impact of these elements is very important to improve the quality of the obtained moving objects' coordinates. The results show that GPS-only solution brings horizontal results several times less accurate than the results obtained by SPP in very challenging surroundings, such as urban areas with tall building and road dense road infrastructure.

Additionally, it has to be considered that during the kinematic positioning the estimation of the height may bring some complications connected with the appropriate stochastic modeling of the zenith troposphere delay. The optimal value of the zenith troposphere delay random walk is hard to determine for the kinematic positioning because of the changes in the receiver's heights. This problem should be considered in the further works. The presented results indicate that the quality possible to obtain during processing supported by 4 systems may bring better results than the GPS-only solution. Simultaneously, users have to be aware of the processing problems including those mentioned in this work.

\section{CONCLUSION}

The conducted works illustrate the potential of the multi-GNSS kinematic SPP and PPP. The processing methods, which have to be developed in the future, should increase the quality possible to obtain with the multi-GNSS constellation. It has to be mentioned that GNSS, such as Galileo and BeiDou, are still being developed and the number of satellites possible to observe still grows. This expansion may influence the position quality improvement in city centers with tall buildings in the near future.

Acknowledgments: This work has been supported by the Polish National Science Centre research Project No.UMO-2014/15/B/ST10/00084 and the Wrocław Center of Networking and Supercomputing (http://www.wcss.wroc.pl/) computational Grant using MATLAB Software License No.: 101979. The authors gratefully acknowledge the International GNSS Service (IGS) for providing multi-GNSS observations, the Centre National d'Études Spatiales (CNES) for providing real-time streams, the Bundesamt für Kartographie und Geodäsie (BKG) for providing the open-source BNC software, the Geotronics Dystrybucja Ltd. (Poland) for providing observation data for OLES permanent station. 


\section{REFERENCES}

Aughey RJ., and Falloon C (2010). Real-time versus post-game GPS data in team sports, Journal of Science and Medicine in Sport, 13, 348-349, doi: 10.1016/j.jsams.2009.01.006

Bisnath S., and Gao Y (2009). Current State of Precise Point Positioning and Future Prospects and Limitations,. In: Observing our Changing Earth. Springer Berlin Heidelberg, Berlin, Heidelberg, pp 615-623

Dow JM., Neilan RE., and Rizos C (2009). The International GNSS Service in a changing landscape of Global Navigation Satellite Systems,. J. Geod. 83:191-198

Geng J., Teferle FN., Meng X., and Dodson AH (2010). Kinematic precise point positioning at remote marine platforms, GPS Solutions, 14, 343-350, doi: 10.1007/s10291-009-0157-9

Groves PD (2011). Shadow matching: A new GNSS positioning technique for urban canyons, Journal of Navigation, 64, 417-430, doi: 10.1017/S0373463311000087

Guo J., Li X., Li Z., Hu L., Yang G., Zhao C., Fairbairn D., Watson D., and Ge M (2018). Multi-GNSS precise point positioning for precision agriculture, Precision Agriculture, 1-17, doi: 10.1007/s11119-018-9563-8

Hadas T (2015). GNSS-Warp Software for Real-Time Precise Point Positioning, Artificial Satellites, 50, 59-76, doi: 10.1515/arsa-2015-0005

Hadas T., and Bosy J (2015). IGS RTS precise orbits and clocks verification and quality degradation over time, GPS Solutions, 19, 93-105, doi: 10.1007/s10291-014-0369-5

Hadas T., Teferle FN., Kazmierski K., Hordyniec P., and Bosy J (2017). Optimum stochastic modeling for GNSS tropospheric delay estimation in real-time, GPS Solutions, 21, 1069-1081, doi: 10.1007/s10291-016-0595-0

He H., Li J., Yang Y., Xu J., Guo H., and Wang A (2014). Performance assessment of singleand dual-frequency BeiDou/GPS single-epoch kinematic positioning, GPS Solutions, 18, 393-403, doi: 10.1007/s10291-013-0339-3

Hernández-Pajares M., Juan JM., Sanz J., Orús R., García-Rodríguez A., and Colombo OL (2004). Wide area real time kinematics with Galileo and GPS signals,. In: 17th International Technical Meeting of the Satellite Division of the Institute of Navigation, ION GNSS 2004. pp 2541-2554

Héroux P., Gao Y., Kouba J., and Lahaye F (2004). Products and applications for Precise Point Positioning-Moving towards real-time,. In: Proc. ION ITM 2004, Institute of Navigation, Long Beach, CA, USA, September 21 - 24,. pp 1832-1843

$\mathrm{Hu}$ Y., Cheng L., and Wang X (2016). Quality analysis of the campaign GPS stations observation in Northeast and North China,. doi: 10.1016/j.geog.2016.03.008

IGS (2015). RTCM-SC104, RINEX-The Receiver Independent Exchange Format (Version $3.03)$, 
Kazmierski K., Hadas T., and Sośnica K (2018a). Weighting of Multi-GNSS Observations in Real-Time Precise Point Positioning, Remote Sensing, 10, 84, doi: 10.3390/rs10010084

Kazmierski K., Sośnica K., and Hadas T (2018b). Quality assessment of multi-GNSS orbits and clocks for real-time precise point positioning, GPS Solutions, 22, 11, doi: 10.1007/s10291-017-0678-6

Knoop VL., de Bakker PF., Tiberius CCJM., and van Arem B (2017). Lane Determination With GPS Precise Point Positioning, IEEE Transactions on Intelligent Transportation Systems, 18, 2503-2513, doi: 10.1109/TITS.2016.2632751

Kouba J (2015). A Guide to using international GNSS Service ( IGS ) Products, Geodetic Survey Division Natural Resources Canada Ottawa, 6, 34

Lachapelle G., Casey M., Eaton M., Kleusberg A., Tranquilla J., and Wells D (1987). GPS Marine Kinematic Positioning Accuracy and Reliability,. In: Proceedings International Symposium on Marine Positioning. Springer Netherlands, Dordrecht, pp 113-147

Laurichesse D (2011). The CNES Real-time PPP with undifferenced integer ambiguity resolution demonstrator,. In: ION GNSS. Portland

Leandro RF., Langley RB., and Santos MC (2008). UNB3m_pack: A neutral atmosphere delay package for radiometric space techniques, GPS Solutions, 12, 65-70, doi: 10.1007/s10291-007-0077-5

Loyer S., Perosanz F., Mercier F., Capdeville H., and Marty J-C (2012). Zero-difference GPS ambiguity resolution at CNES-CLS IGS Analysis Center, Journal of Geodesy, 86, 991-1003, doi: 10.1007/s00190-012-0559-2

Martín A., Anquela AB., Dimas-Pagés A., and Cos-Gayón F (2015). Validation of performance of real-time kinematic PPP. A possible tool for deformation monitoring, Measurement, 69, 95-108, doi: 10.1016/J.MEASUREMENT.2015.03.026

Martinez FG., and Waller P (2009). GNSS clock prediction and integrity,. In: 2009 IEEE International Frequency Control Symposium Joint with the 22nd European Frequency and Time forum. IEEE, pp 1137-1142

Montenbruck O., Steigenberger P., Khachikyan R., Weber G., Langley R., Mervart L., and Hugentobler U (2014). IGS-MGEX Preparing the Ground for Multi-COnstellation GNSS Science, Inside GNSS, 42-49

Paziewski J., Sieradzki R., and Baryla R (2018). Multi-GNSS high-rate RTK, PPP and novel direct phase observation processing method: application to precise dynamic displacement detection, Measurement Science and Technology, 29, 35002, doi: $10.1088 / 1361-6501 / \mathrm{aa} 9 \mathrm{ec} 2$

Petit G., and Luzum B (2010). IERS Conventions, Bureau International Des Poids Et Mesures Sevres (France), ISBN 3-898, 179 
Prange L., Orliac E., Dach R., Arnold D., Beutler G., Schaer S., and Jäggi A (2017). CODE's five-system orbit and clock solution - the challenges of multi-GNSS data analysis, Journal of Geodesy, 91, 345-360, doi: 10.1007/s00190-016-0968-8

Realini E., Caldera S., Pertusini L., and Sampietro D (2017). Precise GNSS Positioning Using Smart Devices, Sensors, 17, 2434, doi: 10.3390/s17102434

Rieke M., Foerster T., Geipel J., and Prinz T (2011). High-precision positioning and real-time data processing of UAV systems, International Archives of the Photogrammetry, Remote Sensing and Spatial Information Sciences, 28, 6, doi: 10.5194/isprsarchives-XXXVIII-1-C22-119-2011

Rietdorf A., Daub C., and Loef P (2006). Precise Positioning in Real-Time using Navigation Satellites and Telecommunication, Procedings of the 3rd Workshop on Positioning, Navigation and Communication, 123-128

Tang X., Roberts GW., Li X., and Hancock CM (2017). Real-time kinematic PPP GPS for structure monitoring applied on the Severn Suspension Bridge, UK, Advances in Space Research, 60, 925-937, doi: 10.1016/J.ASR.2017.05.010

Tayari E., Jamshid AR., and Goodarzi HR (2015). Role of GPS and GIS in precision agriculture, Journal of Scientific Research and Development, 2, 157-162

Teunissen PJG., and Montenbruck O (eds) (2017). Springer Handbook of Global Navigation Satellite Systems,. Springer International Publishing, Cham

Tomoji T (2007). RTKLIB: An open source program package for GNSS positioning,

Wang L., Li Z., Ge M., Neitzel F., Wang Z., and Yuan H (2018). Validation and Assessment of Multi-GNSS Real-Time Precise Point Positioning in Simulated Kinematic Mode Using IGS Real-Time Service, Remote Sensing, 10, 337, doi: 10.3390/rs10020337

Weber G., and Mervart L (2009). The BKG Ntrip Client (BNC),. Mitteilungen des Bundesamtes fuer Kartographie und Geod

Xu G (2016). GPS: Theory, algorithms and applications, Third edit,

Yang F., Li L., Zhao L., and Cheng C (2017). GPS/BDS Real-Time Precise Point Positioning for Kinematic Maritime Positioning,. Springer, Singapore, pp 295-307

Zumberge JF., Heflin MB., Jefferson DC., Watkins MM., and Webb FH (1997). Precise point positioning for the efficient and robust analysis of GPS data from large networks, Journal of Geophysical Research: Solid Earth, 102, 5005-5017, doi: 10.1029/96JB03860

Received: 2018-03-21,

Reviewed: 2018-04-18, by J. Paziewski, and 2018-04-19, by W. Kosek, Accepted: 2018-04-25. 